\title{
EXPERIENCIAS EXITOSAS DE ASOCIATIVIDAD: UN CASO DE EMPODERAMIENTO DE LAS MUJERES RURALES Y EQUIDAD DE GÉNERO EN CADENAS DE VALOR AGRÍCOLA
}

\section{Successful experiences of associativity: a case of empowerment of rural women and gender equity in agricultural value chains.}

Yeny P. Silva-Jiménez¹, Carlos Andrés Durán², Clara Milena Concha ${ }^{3}$, Juan Diego Otero ${ }^{4}$

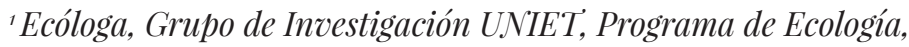
Fundación Universitaria de Popayán, Dirección: Calle 69CN $\# 8 D-03$ Ciudadela San Eduardo. Correo:yenysilva86@gmail.com

${ }^{2}$ Grupo de Investigación UNIET, Programa de Ecología, Fundación Universitaria de Popayán, Correo: carlos.duran@docente.fup.edu.co

${ }^{3}$ Grupo de Investigación en Tecnología y Ambiente - GITA, Corporación Universitaria Autónoma del Cauca. Correo: clara.concha.l@uniautonoma.edu.co

${ }^{4}$ SENA. Popayán (Colombia) Correo: juandote@gmail.com

\section{Resumen}

Las mujeres rurales se encuentran en una situación de desventaja para acceder a recursos productivos y reconocimiento como productoras, proveedoras y compradoras a lo largo de las cadenas agroalimentarias. El objetivo de esta investigación fue analizar las alternativas de empoderamiento de las mujeres
Historia del artículo

Fecha de recepción:

$31 / 10 / 2020$

Fecha de aceptación:

$01 / 12 / 2020$ 
rurales e integración de equidad de género en cadenas de valor agrícola a partir de un caso de estudio de asociatividad en la Vereda San Roque (Sotará, Cauca). Para este estudio de caso se hizo el análisis de cadenas de valor a nivel macro, meso y micro, asimismo, se empleó la matriz de priorización para la toma de decisiones, como método de valoración de alternativas de empoderamiento femenino. Los resultados se confrontaron con el contexto sociocultural, político y económico en un estudio de caso. Se obtuvo que las cadenas de valor agrícola requieren ser intervenidas desde una perspectiva de género, que promueva el empoderamiento de las mujeres como productoras y que incentive su participación escalonada a lo largo de las cadenas de valor. Se presentan doce alternativas de empoderamiento que buscan mejorar la posición de las mujeres en las cadenas implementando procesos agroecológicos, transformando los roles tradicionales de las mujeres en su comunidad, pues permite desarrollar habilidades, adquirir destrezas, fortalecer conocimientos y gestionar procesos que aporten valor, para acceder a múltiples espacios, oportunidades y beneficios.

Palabras Claves: Empoderamiento femenino, equidad de género, agroecología, desarrollo rural.

\section{Abstract}

Rural women are at a disadvantage to access productive resources and recognition as producers, suppliers and buyers throughout the agri-food chains. The objective of this research was to analyze the alternatives of empowerment of rural women and integration of gender equity in agricultural value chains from a case study of associativity in the San Roque village (Sotará, Cauca). For this case study, the analysis of value chains was carried out at the macro, meso and micro levels, likewise, the prioritization matrix was used for decision-making, as a method for evaluating alternatives for female empowerment, the results were compared with the sociocultural, political and economic context in a case study. It was found that agricultural value chains need to be intervened from a gender perspective, that promotes the empowerment of women as producers and that encourages their staggered participation along the value chains. Twelve empowerment alternatives are presented that seek to improve the position of women in the chains by implementing agroecological processes, transforming the traditional roles of women in their community, since it allows them to develop skills, acquire skills, strengthen knowledge and manage processes that add value, to access multiple spaces, opportunities and benefits.

Keywords: Female empowerment, gender equality, agroecology, rural development.

\section{Introducción}

La tendencia globalizada de mercados ha provocado modificaciones en el sector agrícola y alimentario, dando apertura a nuevas oportunidades de comercialización y la creación de fuertes vínculos entre grandes productores y amplios mercados. Sin embargo, las reformas agrarias han generado 
a los pequeños productores, obstáculos al momento de beneficiarse del comercio nacional e internacional (ONU, 2017; FAO, 2013). En este sentido, las mujeres han encontrado más dificultades, comparado con los hombres, para acceder a recursos productivos y participar en igual medida en las cadenas de valor agroalimentarias, pese a que ellas representan el $43 \%$ de la mano de obra agrícola y participan intensamente en la producción de alimentos y cultivos comerciales en todo el mundo (FAO, 2017).

En Latinoamérica, las mujeres rurales representan el pilar productivo del sector agropecuario, ya que son con la mitad de la fuerza laboral, no obstante, tienen menos acceso que los hombres a los activos productivos y a servicios financieros (Nobre et al., 2017). Por su parte, la mujer del sector rural colombiano ha afrontado históricamente un alto índice de desigualdad social y económica, debido a las dificultades que el sistema patriarcal impone para acceder a los factores de producción, principalmente la tierra (Cediel y Morales, 2019), pues la mujer no tiene liderazgo, autoridad ni control de la propiedad. Según, la ONG Comisión Colombiana de Juristas (2011), las políticas de Estado, la legislación colombianay la ausencia de reconocimiento social de las mujeres rurales, obstaculiza garantizar el derecho de titularidad de predios y ejercer propiedad sobre la tierra, propiciando un alto nivel de vulnerabilidad en cadenas de valor agrícola. Aun cuando se dedican a múltiples actividades de la produccióny mantenimiento de cultivos, no son registradas como productoras, proveedoras y compradoras directas, sino que su trabajo se considera parte de la "ayuda familiar", corroborando que prevalecen desigualdades de género cuyo impacto afecta la calidad de vida de las mujeres y sus familias (Agüero et al., 2012).

En términos de justicia social, a pesar de que los derechos humanos son herramientas para reclamarla, su defensa en función del género se caracteriza por la violación de los derechos de las mujeres, esto trae como consecuencia su desempoderamiento y menor capacidad de acción, incidiendo negativamente en la toma decisiones que mejoren sus medios de vida (KIT AgriProFocus e IIRR, 2013). La discriminación por motivo de género trae como consecuencia una distribución inequitativa de las ventajas, activos y oportunidades para las mujeres, enfrentando barreras culturales que provocan su marginación en educación, trabajo y remuneración. Las mujeres que participan en el engranaje del desarrollo rural, tienen poca autoconciencia y valoración de su rol productivo en sus familias, comunidades y a lo largo de las cadenas de valor (Cortínez, 2016).

Dentro de este marco, es necesario plantear un nuevo modelo de desarrollo rural, que dialogue con la viday los derechos humanos, con mejor uso y acceso a la tierra, así como a otros recursos productivos, mediante la necesaria redistribución equitativa con enfoque de género. De esta forma se dignifican y visibilizan los escenarios donde la mujer reconoce su identidad, poder y capacidad para contribuir a la sostenibilidad de la casa común y en la reducción de la pobreza en territorios rurales (Cediel y Morales, 2019). Esto, sin duda, aporta con 
acciones positivas para hombres y mujeres que permitan integrar la igualdad en las estructuras y organizaciones sociopolíticas, para dotar a las mujeres de un papel protagónico mediante el empoderamiento económico y myor participación en la toma de decisiones de todos los ámbitos de la vida social (Duarte y García, 2016).

En este escenario, se plantea el siguiente estudio de caso, cuyo objetivo es analizar las alternativas de empoderamiento e integración de criterios de equidad de género en el fortalecimiento de cadenas de valor agrícola a través de la experiencia exitosa de asociatividad en mujeres de la vereda San Roque del municipio de Sotará, quienes, desde lo local, han ganado espacio y participación en las dinámicas productivas, socioculturales y políticas del sector rural. Tras este análisis se plantean alternativas de empoderamiento y la importancia de la equidad de género para el desarrollo rural.

\section{Materiales y Métodos}

\section{Área de estudio}

La vereda San Roque, departamento del Cauca (Colombia), está ubicada en la parte noroccidental del corregimiento de Chapa a $15 \mathrm{~km}$ aproximadamente, vía al sur de Paispamba, cabecera del municipio de Sotará. Se localiza en las coordenadas $02^{\circ} 13$ latitud Norte y $76^{\circ} 38$ longitud Oeste (Fig. 1). Con una altitud desde los 2300 msnm hasta los 4400 msnm aproximadamente y una temperatura promedio de $13^{\circ} \mathrm{C}$. Limita al Norte con la vereda El llano de Sotará, al Sur con la vereda
El Diviso, al Oriente con la vereda Chapa y al Occidente con la vereda Piedra de León. Su acceso es por vía terrestre y la ruta más

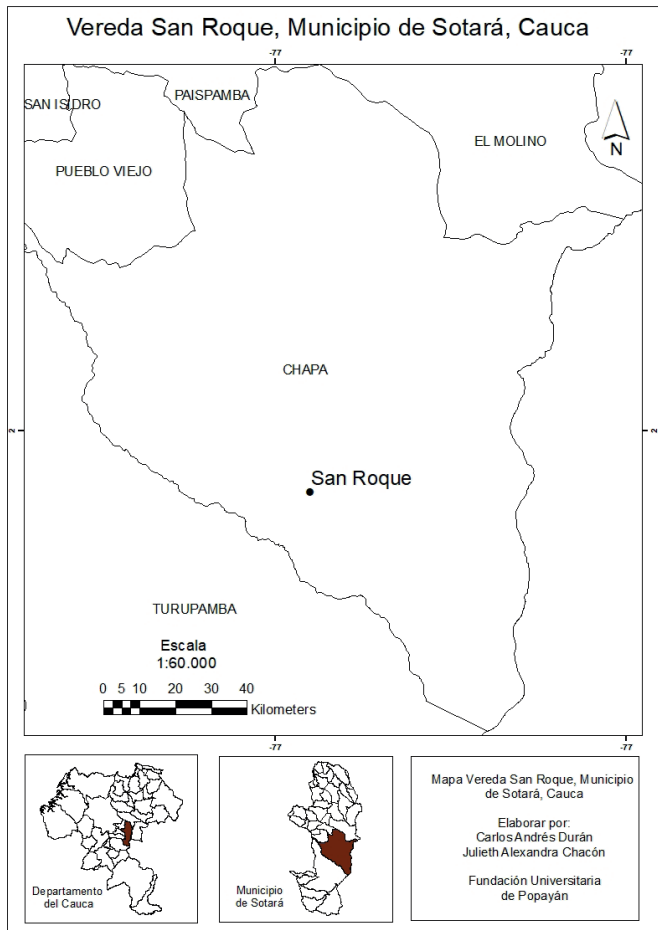

Figura 1. Mapa vereda San Roque (Fuente: elaboración propia).

concurrida es Popayán, Timbío, Paispamba, Chapa, con un recorrido de $41 \mathrm{~km}$.

Históricamente, la zona ha sido un escenario dominado por terratenientes, que aprovechaban la mano de obra de campesinos para la tala de bosques y la formación de potreros para la explotación ganadera. Algunos predios fueron heredados por generaciones; otros fueron adquiridos por los campesinos de la región, luego de graves problemas de orden público y su 
posterior venta. Es así como este sector dejó de pertenecer a la vereda Chapa, para convertirse en la vereda San Roque, fundada en 1985, tiempo en el que se otorga la Personería Jurídica a la primer Junta de Acción Comunal, por los campesinos agrupados en 55 familias, quienes ya venían trabajando en su organización comunitaria, con objetivos de beneficio común, como por ejemplo, la construcción de una vía de acceso al territorio.

Actualmente, habitan 35 familias de máximo cuatro integrantes cada una, muchas personas salieron de la vereda $\mathrm{e}$ incluso del municipio para buscar mejores condiciones de vida e ingresos económicos. Estas familias campesinas conviven con un grupo de indígenas Yanacona, proveniente del Resguardo de Río Blanco, quienes en su llamado "proceso de recuperación de tierras" invadieron una finca de gran extensión en 2002, la cual fue entregada posteriormente por parte del gobierno, junto con dos predios más. Por esto, la población mayoritaria en el territorio es indígena, agrupada en 72 familias, con las cuales poco a poco se han ido superando dificultades relacionadas con el sistema de organización, cosmovisión y derechos.

Aunque los procesos de desarrollo comunitario se dan de manera independiente, la desigualdad de género en función del trabajo rural es común en ambas. Los hombres se dedican al trabajo pesado, comercializan lo que producen, generan los recursos económicos de sobrevivencia y, por consiguiente, deciden como se gastan, además, son poseedores de la tierra. Por su parte, las mujeres se encargan de las labores del hogar, cuidado de animales (ganado, gallinas) y cultivos de pan coger, sin recibir reconocimiento social ni económico por su labor. Esta desigualdad responde a que el dinero que se percibe se gasta de acuerdo a lo que los hombres consideran importante, sin tener en cuenta las necesidades de las mujeres. Esto evidencia, la desigualdad social y económica, la carencia de participación de las mujeres en la toma de decisiones, la vulnerabilidad social y bajo nivel de empoderamiento.

\section{Métodos}

La presente investigación cualitativa es un estudio de caso (Martínez, 2011), con el cual se busca analizar desde la experiencia de la Asociación de Mujeres Emprendedoras de San Roque ASMESAR, las alternativas de empoderamiento de mujeres rurales y la incorporación de criterios de equidad de género en el fortalecimiento de cadenas de valor agrícola. Para ello, la revisión de literatura se hizo mediante la síntesis de resultados encontrados en investigaciones cualitativas, aplicando criterios del marco de referencia Search, AppraisaL, Synthesis y Analysis (SALSA), propuesto por Grant \& Booth (2009), centrado en aspectos económicos, políticos y socioculturales con perspectiva de género en las cadenas de valor agrícola.

Como método de recolección de información primaria se recurrió a herramientas proporcionadas por métodos participativos (Durston y Miranda. 2002), lo que permitió el diálogo de saberes (Toledo, 2011), para abordar de forma cercana la experiencia de 
asociatividad, facilitando la comprensión y apropiación de conocimientos compartidos, así como, la identificación de costumbres, experiencias y lecciones aprendidas de las mujeres emprendedoras en busca de su propósito y las interacciones con entidades gubernamentales y privadas asociadas a la zona.

El análisis de la cadena de valor agrícola desde una perspectiva de género, se desarrolló a través de la recolección de datos para análisis de cadenas de valor en los niveles macro, meso y micro (Terrillon, 2010). Por medio de listas de preguntas de control, permite comprender roles de género, acceso a recursos, control sobre los beneficios e influencia sobre los factores habilitadores. Como resultado se obtiene la identificación de limitaciones y oportunidades para el empoderamiento de las mujeres rurales. En el nivel superior se consideró el entorno institucional y las interrelaciones entre actores en toda la cadena, analizando si son favorables al desarrollo de pequeños productores, siendo equitativas, inclusivas y sensibles al género. El análisis de nivel medio abordó la sensibilidad de género en instituciones y organizaciones locales, en cuanto a cómo aplican los principios de equidad de género estructural y culturalmente, en la forma en que se garantizan los servicios. En el nivel inferior, se identificaron restricciones que enfrentan las mujeres en el entorno del hogary que repercuten en los niveles superiores.

La selección de alternativas de empoderamiento femenino y fortalecimiento de las cadenas de valor agrícola se realizó mediante la Matriz de Priorización para la Toma de Decisiones (Medina et al., 2010), la cual permite seleccionar las alternativas estratégicas que fortalezcan las cadenas de valor agrícola y enfocar los recursos para el empoderamiento de las mujeres rurales. Se compone de alternativas planteadas desde cuatro dimensiones: tradición; equidad de género; cambio; estándares, certificación y etiquetas, las cuales fueron contrastadas con las variables agencia, estructura, desarrollo y gobernabilidad de la cadena. La calificación se hizo en una escala de puntuación de 1 a 5; donde, 1 es Inexistente, 2 es Insuficiente, 3 es Regular, 4 es Bueno y 5 es Excelente, los cuales indican la condición de las mujeres rurales frente a la variable para favorecer la implementación de la alternativa. Dicha matriz fue adaptada al estudio tomando como referencia la Matriz de empoderamiento de la cadena desarrollada por KIT, Agri-ProFocus and IIRR, (2013).

\section{Resultados y discusión}

Producto del proceso de investigación, se presentan los aportes que han surgido en torno al desarrollo de cadenas de valor sensibles al género en cuatro secciones. La primera expone las experiencias exitosas de mujeres rurales al enfrentarse a retos y aprovechar oportunidades para adelantar procesos asociativos, así como el resultado de inspirar su empoderamiento en procesos agroecológicos. En segunda instancia, se presenta el análisis de la cadena de valor agrícola con perspectiva de género y posteriormente se prioriza 
las alternativas de empoderamiento de las mujeres rurales para el fortalecimiento de esta. Por último, se muestran los principales hallazgos y reflexiones surgidas en torno a la importancia de incorporar criterios de equidad de género en cadenas de valor agrícola y su contribución al desarrollo rural y la sostenibilidad ambiental del territorio.

\section{Experiencias exitosas de asociatividad un camino al empoderamiento de la mujer rural}

Las actividades realizadas por organizaciones que agrupan productores dentro de una figura jurídica, que favorecen la producción, transformación y comercialización de productos en busca del beneficio colectivo, debe considerarse como un proceso exitoso de asociatividad, ya que el resultado no solo es la generación de ingresos económicos, sino que también promueve una serie de interrelaciones sociales, institucionales, políticas y financieras, convenientes para el empoderamiento del productor y el desarrollo rural (Mamani, 2017). Así quedó comprobado, con la experiencia de dos asociaciones de mujeres que a partir de adelantar procesos asociativos y agroecológicos encontraron oportunidades para su empoderamiento.

\subsection{Desarrollo de procesos asociativos}

La Asociación de Mujeres Emprendedoras de San Roque ASMESAR, del municipio de Sotará (Cauca), surge por la necesidad de una mujer, CeciliaJiménez, por transformar su realidad económica y la de su entorno social. Así fue tocando puertas a entidades gubernamentales para lograr adherirse a un programa que ofreciera oportunidades para la región. Ella buscó la forma de canalizar recursos del gobierno, ya que, en su recorrido la respuesta había sido que:

"si existía la posibilidad de obtener algún beneficio, solo sí, esa petición era presentada por un grupo de campesinos que conformaran una asociación" (C. Jiménez, 26 mayo de 2020).

De ahí surgió la idea de convocar a las mujeres de las veredas que integran el corregimiento de Chapa a unirse y trabajar en torno a un beneficio común.

Este proceso no ha sido fácil, pues, según lo expuesto por la lideresa, hasta ese momento no había experiencias de asociatividad en la zona, no se sabía cómo adelantar tal requisito y no se contaba con el conocimiento para conformar la asociación. Aunque muchas aceptaron reunirse y escucharla, pocas se interesaron, algunas mujeres motivadas por trabajar de verdad, otras interesadas en recibir alguna ayuda temporal y otras sencillamente por no quedarse atrás, conformaron ASMESAR. Una vez concretadas las personas, fue doña Cecilia quien tocó puertas en las entidades para pedir capacitación y acompañamiento en la creación de la organización de mujeres.

La lideresa relata que en su territorio vivían bajo el rol tradicional de la mujer rural, donde quien tiene y decide es el poseedor de la tierra, en ese caso, los esposos. Cecilia motivó a sus vecinas y consultó con personas que la pudieran asesorar en la 
documentación hasta lograr su cometido. Confiesa que no fue fácil y que enfrentó varios obstáculos y limitaciones, como falta de conocimientos, creencias y tradiciones sociales respecto al acceso a recursos.

ASMESAR es una asociación conformada por quince mujeres emprendedoras, cinco de ellas son las más activas y han mejorado su posición en la realidad social rural, dedicándose al fortalecimiento de cada unidad productiva donde cultivan mora y tomate de cocina, también dan valor agregado a sus productos, ofreciendo mermeladas, salsas y combinaciones con derivados lácteos (Fig. 2). Sin embargo, hay dificultades en la disposición de las asociadas para continuar en el proceso por limitaciones como las barreras impuestas por el rol tradicional de la mujer rural, el cual les da poca capacidad de decisión, sumado a la sobrecarga laboral, poca disponibilidad de tiempo y dificultad de acceso a recursos económicos, información, mercados y posibilidades de comercialización de sus productos.

Las mujeres rurales que participan de procesos asociativos han visto la oportunidad de cambiar su realidad y han alcanzado cierto nivel de empoderamiento, buscando que la actividad productiva motive

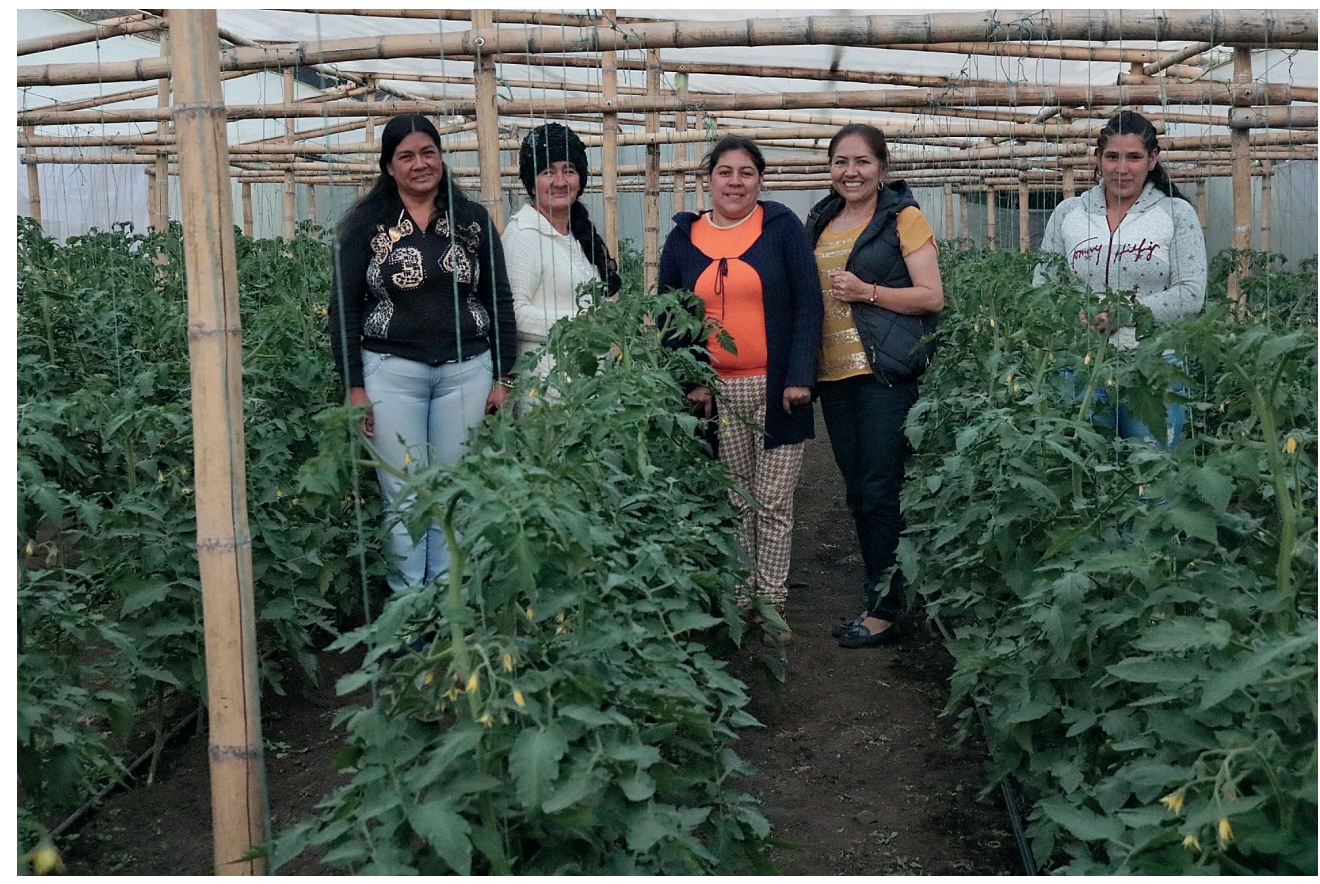

Figura 2. Integrantes de ASMESAR (Fuente: esta investigación). 
la participación activa de todas las asociadas y les permita mejorar su economía, al tiempo que promueven iniciativas de desarrollo para su vereda. Además de buscar una producción sostenible en el entorno de la organización comunitaria, aprovechar adecuadamente los recursos naturales y asegurar la interacción armónica entre la comunidad y el ambiente.

No obstante, también se refleja que a pesar de los avances aún falta mucho por recorrer, puesto que se evidencia poca presencia institucional, y la carencia de políticas públicas, planes y programas que verdaderamente creen oportunidades para las mujeres rurales, las cuales garanticen espacios de participación, información, capacitación y fortalecimiento de sus capacidades, desarrollo de habilidades e implementación de actividades productivas a partir de sus conocimientos tradicionales, pero que verdaderamente fortalezcan el proceso de empoderamiento femenino y económico, que sea sostenible y replicador de experiencias de éxito.

\subsection{Desarrollo de procesos agroecológicos.}

Según Sicard (2010) la agroecología se define como la ciencia que estudia la estructura y función de los agroecosistemas desde las relaciones ecológicas y culturales, entendiendo por cultural, aspectos históricos, socioeconómicos, técnicos, políticos e institucionales que inciden en el territorio productivo. Reconocer y analizar de forma integral estas relaciones, facilita la articulación de actividades dentro de los agroecosistemas a fin de armonizar su producción con la conservación. Esto requiere de acción participativa, implementación de saberes y recursos autóctonos, salud ambiental y la diversificación, para lograr eficiencia energética, aprovechamiento de los ciclos naturales y finalmente, prescindir de insumos externos (Segovia, 2009).

Desde esta perspectiva, la agroecología plantea un camino de empoderamiento femenino en el sector rural, ya que abre espacios de participación para mujeres, favoreciendo las relaciones de género y brindando la oportunidad de diferenciación como productoras, realizar su venta directa y generar ingresos económicos (Zuluaga, Catagora y Siliprandi. 2018). Además, motiva la integración, el intercambio de saberes, el cambio de rutinas, la generación de oportunidades para aprender, interactuar y mejorar las expectativas de vida. Estas acciones motivan cambios, generan movimiento e inquietud en las mujeres, creando la necesidad de empoderarse y asumir una nueva actitud como tomadoras de decisiones ante las responsabilidades que histórica y culturalmente han llevado sobre sus hombros sin merecido reconocimiento.

La agroecología, se puede entender como la respuesta a las discusiones en materia de agricultura y desarrollo rural como la mejor alternativa para conciliar la conservación del ambiente con aspiraciones humanas, como es el empoderamientoy satisfacción de necesidades de las mujeres rurales (Segovia, 2009). Desde la perspectiva de Altieri (1999) esta disciplina, provee los principios ecológicos básicos para estudiar, diseñar y manejar agroecosistemas 
productivos, que conserven el recurso natural, además de ser culturalmente sensibles, socialmente justos y económicamente viables.

Otras experiencias como la Asociación de Mujeres Campesinas de Matanza ASOCIMUCAM y Asociación de Mujeres Unidas Construyendo Futuro ASOCIMUCOF de la provincia de Soto Norte (Santander, Colombia), ratifican que, a partir del empoderamiento personal y económico que adquieren a través de su trabajo asociativo y la creación de cultivos de pancoger, también han logran una mejora en la calidad de vida de las asociadas y sus familias (Díaz-Pérez y Silva-Niño, 2019). Como resultado de esta actividad tradicional, se promueve el autoconsumo para garantizar la seguridad alimentaria de las familias; con la adecuada gestión colectiva de los excedentes producidos en las huertas, se promueve la generación de ingresos económicos para las mujeres.

A través de la constitución de una red de comercialización de productos agroecológicos, se fomenta la participación activa de las asociadas, motivándolas a conquistar espacios anteriormente cerrados para las mujeres y disminuyendo la brecha de desigualdad. Así mismo, ha aumentado su interés por auto cuidarse, ejercer el control de su vida y transformar la visión de actividad tradicional. Lo cual las ha llevado a desarrollar su liderazgo, capacidad de acción y tomar decisiones para derribar limitaciones, apropiarse de los derechos de la mujer y acceder a nuevas oportunidades de crecimiento personal, familiar y económico.
A nivel comunitario, ha permitido el empoderamiento femenino en la zona, facilitando el acceso a recursos técnicos y económicos para desarrollar emprendimientos. Igualmente, se ha promovido la recuperación de saberes tradicionales enfocados a la producción agroecológica, como alternativa para garantizar la soberanía alimentaria. Además, ha generado mayor consciencia en la protección y conservación de la biodiversidad, interesándose en la recuperación e intercambio de semillas autóctonas, el escalonamiento de la producción de autoconsumo y la sostenibilidad ambiental.

\section{Análisis de la cadena de valor agrícola}

En la cadena de valor agrícola, las mujeres rurales se dedican a múltiples actividades, como la siembra, trasplante, cosecha, producción y mantenimiento de cultivos; aun así, no son registradas como productoras directas (Agüero, 2012). Tal desconocimiento, genera sistemáticamente menos probabilidades de adquirir tierra y acceder a insumos agrícolas, bajo el prejuicio de que la mujer no debe poseer la tierra en propiedad, facilitando que estos recursos estén más disponibles para el hombre (Zarate, 2019). Ante ello, el respaldo del Estado es mínimo o casi nulo, dado que hasta 2010, la gestión para la entrega de títulos de predios se limitó a la convocatoria para aspirar a un subsidio (ONG Comisión Colombiana de Juristas, 2011). A esto se suma la escasa formación educativa de las mujeres rurales y poco acceso a la información, enfrentando mayores dificultades para alcanzar un protagonismo en las decisiones 
productivas y tecnológicas, acceder a los beneficios potenciales de la cadena de valor, a las nuevas tecnologías y a los instrumentos de política pública que se diseñan para promover las innovaciones (Terrillon, 2010).

Algo semejante ocurre con el acceso a mercados y oportunidades de comercialización, puesto que la restricción de espacios organizativos para las mujeres, dada la marcada división de los roles de género, limitan el acceso a las redes que pueden proporcionarles información. Además, su capital social a menudo se basa en la familia y los vecinos, mientras que los hombres incluyen a compañeros de trabajo y otros contactos empresariales. En consecuencia, las mujeres tienen menor probabilidad de beneficiarse económicamente de sus relaciones sociales (Kim y Sherraden, 2014).

Las mujeres rurales son las encargadas de las labores domésticas y el cuidado de los niños, así como de otras actividades agrícolas relacionadas con el cultivo de productos alimentarios (FAO, 2014). Esto reduce considerablemente su productividad, ya que soportan una enorme carga de trabajo que no les permite hacer control de su tiempo, invirtiéndolo en la producción, labores domésticas, y actividades productivas adicionales. Asimismo, las mujeres siguen siendo responsables de garantizar la seguridad alimentaria y nutricional en sus hogares: esto incluye el cultivo de productos alimentarios al igual que la preparación de alimentos (Acosta y Zambrano, 2017).

Los resultados que se relacionan según las causas de fondo y problemática de género en la cadena de valor agrícola, los cuales son analizados en los niveles macro, meso y micro para identificar las limitaciones y oportunidades de las mujeres rurales, finalmente se determinan las acciones para intervenir de forma adecuada (Tabla 1).

Según este análisis de la matriz a nivel macro, meso y micro de las cadenas de valor agrícola, la mayor parte de las mujeres empresarias rurales tienen limitaciones como el manejo técnico para la elaboración, acabado y presentación de sus productos; el poco reconocimiento y valoración de su trabajo y aporte a la seguridad alimentaria; así como la baja autoestima que aún persiste en muchas de ellas (Agüero, 2012). A esta situación se suma la limitación de acceso a los servicios financieros para el desarrollo de sus iniciativas, el escaso manejo y conocimiento para la gestión, canales de comercialización, administración empresarial, y poco equipamiento para el desarrollo de su trabajo. Además, los reducidos ingresos que perciben las mujeres mediante estas actividades en sus propios terrenos o en otras fincas como empleos adicionales, van destinados a sus hogares para cubrir gastos familiares (Moreno, 2015).

Estas limitaciones tienen un efecto importante en la cadena de valor agrícola, dado que las mujeres no tienen disponibilidad de tiempo suficiente para invertirlo en la producción de cultivos. En muchas ocasiones, no pueden cosechar cuando los precios del mercado son más favorables, en cambio lo hacen cuando empieza la temporada escolar y los gastos domésticos son más altos, por lo que reciben 
Tabla 1. Análisis a nivel macro, meso y micro de la cadena de valor agrícola

\begin{tabular}{|c|c|c|c|c|}
\hline & Roles de género & $\begin{array}{l}\text { Acceso a recursos } \\
\text { por género }\end{array}$ & $\begin{array}{c}\text { Control de los } \\
\text { beneficios por } \\
\text { género }\end{array}$ & $\begin{array}{l}\text { Influencia del } \\
\text { género sobre } \\
\text { los factores } \\
\text { habilitadores }\end{array}$ \\
\hline $\begin{array}{l}\text { Nivel } \\
\text { macro }\end{array}$ & $\begin{array}{l}\text { Las mujeres } \\
\text { representan el } \\
43 \% \text { de la mano de } \\
\text { obra y participan } \\
\text { intensamente en } \\
\text { la producción } \\
\text { agrícola. }\end{array}$ & $\begin{array}{c}\text { Las políticas, } \\
\text { programas y } \\
\text { proyectos de fomento } \\
\text { económico en la } \\
\text { cadena de valor } \\
\text { agrícola no favorecen } \\
\text { el acceso de las } \\
\text { mujeres a activos, ni } \\
\text { protegen a las que } \\
\text { han logrado acceder } \\
\text { a ellos. }\end{array}$ & $\begin{array}{c}\text { Bajo } \\
\text { reconocimiento } \\
\text { social y } \\
\text { económico de } \\
\text { las mujeres } \\
\text { como el pilar del } \\
\text { sector agrícola, } \\
\text { la mano de obra } \\
\text { y los sistemas } \\
\text { alimentarios. }\end{array}$ & $\begin{array}{c}\text { Bajo nivel de } \\
\text { participación } \\
\text { de las mujeres } \\
\text { en asociaciones } \\
\text { comunitarias y } \\
\text { organizaciones } \\
\text { gubernamentales. }\end{array}$ \\
\hline $\begin{array}{l}\text { Nivel } \\
\text { meso }\end{array}$ & $\begin{array}{l}\text { Las mujeres no } \\
\text { son reconocidas } \\
\text { como productoras } \\
\text { directas, su trabajo } \\
\text { se considera } \\
\text { parte de la } \\
\text { "ayuda familiar". }\end{array}$ & $\begin{array}{c}\text { Derechos de } \\
\text { propiedad inseguros } \\
\text { o incompletos, } \\
\text { servicios financieros } \\
\text { poco sólidos e } \\
\text { infraestructura } \\
\text { deficiente. }\end{array}$ & $\begin{array}{l}\text { Espacios de } \\
\text { participación } \\
\text { definidos para las } \\
\text { mujeres, pero es } \\
\text { restringidos por } \\
\text { el acceso a los } \\
\text { recursos. }\end{array}$ & $\begin{array}{l}\text { Limitación a } \\
\text { las mujeres } \\
\text { para participar } \\
\text { activamente } \\
\text { en la toma de } \\
\text { decisiones y } \\
\text { desempeñar roles } \\
\text { de importancia } \\
\text { según sus méritos } \\
\text { y capacidades. }\end{array}$ \\
\hline $\begin{array}{l}\text { Nivel } \\
\text { micro }\end{array}$ & $\begin{array}{l}\text { Las mujeres se } \\
\text { encargan de las } \\
\text { labores domésticas } \\
\text { y el cuidado } \\
\text { infantil, además } \\
\text { de actividades } \\
\text { agrícolas } \\
\text { relacionadas } \\
\text { con el cultivo } \\
\text { de productos } \\
\text { alimentarios. No se } \\
\text { reconoce el valor } \\
\text { de estas labores y } \\
\text { su impacto sobre } \\
\text { el tejido social de } \\
\text { las comunidades. }\end{array}$ & $\begin{array}{c}\text { Limitación de } \\
\text { recursos propios, } \\
\text { control de activos } \\
\text { y participación en } \\
\text { las decisiones que } \\
\text { afectan sus vidas y su } \\
\text { entorno. }\end{array}$ & $\begin{array}{c}\text { Dificultad de } \\
\text { acceso a grandes } \\
\text { mercados } \\
\text { y precios } \\
\text { favorables a causa } \\
\text { de la sobrecarga } \\
\text { laboral de las } \\
\text { mujeres, baja } \\
\text { participación } \\
\text { en toma de } \\
\text { decisiones } \\
\text { y manejo de } \\
\text { ingresos. }\end{array}$ & $\begin{array}{c}\text { Bajo nivel de } \\
\text { conciencia y } \\
\text { autovaloración de } \\
\text { su rol productivo } \\
\text { dentro de la } \\
\text { cadena de valor. }\end{array}$ \\
\hline
\end{tabular}

Fuente: elaboración propia. 
menos dinero por su producto. Sumado a esto, las condiciones de vivienda, acceso a la educación y a la información privan a la mujer de oportunidades para potencializar sus habilidades, desarrollarse como persona e incrementar su productividad (Rodríguez, 2018).

La brecha de género está muy marcada en el área rural, los roles de género se imponen sobre las personas, siendo el hogar donde más se acentúan las disparidades de género, dada la asignación inequitativa de los recursos. De igual manera, las dinámicas de los individuos y del hogar, la confianza en sí mismas y la distribución en la toma de decisiones limitan a las mujeres para ejercer poder y capacidad de acción impidiendo su participación y la capacidad para beneficiarse por igual de la cadena de valor agrícola (Palomar, 2016).

La integración de las mujeres rurales a las cadenas de valor agrícola en el nivel de productoras es fundamental y puede considerarse un capital de seguridad, es decir que, la agricultura se convierte en una fuente de alimentación y de ingresos, pues el excedente de producción sirve para la venta. El resultado de pertenecer a la cadena facilita la orientación del proceso productivo, garantiza la comercialización y reduce su vulnerabilidad económica, a pesar de que los productos estén sujetos a las políticas de agricultura y los precios del mercado afecten el poder adquisitivo y los patrones de compra (Zarate, 2019).

Sin embargo, el fin último no es solo el reconocimiento a las mujeres como productoras de la cadena, pues se reduciría a las preocupaciones por preparar la tierra, plantar semillas, aplicar fertilizantes, controlar la fauna y flora circundante y cosechar. En cambio, la perspectiva agroecológica entre los productores que se vinculan en las cadenas agrícolas, además de lograr una producción que respecte los ecosistemas, contribuye significativamente al empoderamiento de las mujeres rurales, involucrándolas en actividades como la selección, clasificación, procesamiento y comercialización de su producción (KIT, Agri-ProFocus and IIRR, 2013).

\section{Empoderamiento femenino en cadena de valor agrícola}

Los agricultores dedicados exclusivamente a la producción son actores básicos en la cadena, a medida que desarrollan más actividades dentro de la misma y se involucran cada vez más en su gobernabilidad, adquieren la oportunidad de optimizar su posición agregando valor (KIT, Agri-ProFocus and IIRR, 2013). Por consiguiente, pueden llegar a desempeñar el rol de socios de la cadena, cuando establecen acuerdos de colaboración sobre intereses compartidos y crecimiento mutuo. Estas alianzas a largo plazo los llevan a convertirse en copropietarios de la cadena, sí se apropian de su actividad y rol dentro de esta y crean empresa.

Por consiguiente, se estaría hablando de empoderamiento de la cadena agrícola y control del productor sobre el valor, en términos de desarrollo y gobernabilidad. 
Sin embargo, este proceso debe ser más equitativo yjusto. Para lograrlo, es importante fortalecerlo desde la perspectiva de género, es decir, contemplar el grupo familiar del productory reconocer sus capacidades y sus aportes en las actividades de la cadena (KIT, Agri-ProFocus and IIRR, 2013).

Por ello, este estudio de caso incorpora dos variables a la matriz de empoderamiento propuesta por KIT, MaLi, and IIRR (2006): agencia y estructura, las cuales hacen referencia a las capacidades de las mujeres y ámbitos institucionales. Según Senders (2015), agencia, se refiere al nivel de empoderamiento $\mathrm{y}$ autoconsciencia de las productoras; entre tanto, estructura, hace referencia al grado en el cual las estructuras de los niveles familiar, comunitario y de la sociedad, se transforman a favor de igualdad de género. Lo anterior también hizo replantear el concepto de desarrollo de la cadena, y entenderlo como el punto de mayor generación de valor agregado por las mujeres (productividad y/ o calidad superior del producto). Así mismo, gobernabilidad de la cadena, se asume como el grado de gestión en la cadena de valor que involucran a las mujeres rurales respecto al control y acceso sobre los recursos.

Si se entiende el empoderamiento como, el proceso mediante el cual se adquiere la capacidad de adoptary participar en la toma de decisiones, cuando esta posibilidad ha sido negada o desconocida (Güiza et al., 2016). Este no puede ser conferido por otro, sino que debe generarse por autodeterminación y por la capacidad de definir metas propias y actuar en consecuencia. Para ello, se hace necesario adelantar acciones que ayuden a superar obstáculos culturales, políticos y económicos que por siglos han provocado desigualdad de oportunidades entre hombres y mujeres para ampliar sus capacidades (Marmolejo, Godoy y González, 2017).

En la siguiente matriz de priorización propuesta por Medina (2010), se presenta una serie de alternativas que para la elaboración de esta investigación fueron puestas a consideración dada su pertinencia y objetivo de fortalecer las cadenas de valor agrícola y empoderar a las mujeres rurales (Tabla 2). Estas se agrupan en cuatro dimensiones identificadas en la dinámica de las cadenas productivas y la realidad de las productoras del campo, tales como tradición, espacios, organización y certificaciones; de las cuales, las de mayor puntuación indican su nivel de contribución en el empoderamiento de la mujer rural (Senders, et al. 2015).

En primer lugar, se presenta la dimensión "Mitigar la resistencia a partir de la tradición", es decir adelantar acciones a partir de los roles tradicionales (cultivos o productos) que las mujeres rurales vienen desempeñando, con el fin de profesionalizar sus tareas cotidianas y darles visibilidad dentro de las cadenas de valor. Con ello, se evita la prevención de las mujeres por miedo al riesgo y el temor de los hombres a perder poder (Senders, et al. 2015). Esta dimensión contempla cuatro alternativas, de las cuales la de mayor calificación es "mejorar la participación de las mujeres por medio de procesos agroecológicos". Posteriormente, la alternativa de "formalizar las cadenas de valor femeninas" y por último se califican las alternativas "convertir responsabilidades 
Tabla 2: Matriz de priorización de alternativas

\begin{tabular}{|c|c|c|c|c|c|}
\hline \multirow{3}{*}{$\begin{array}{l}\text { ALTERNATIVAS DE } \\
\text { EMPODERAMIENTO }\end{array}$} & \multicolumn{5}{|c|}{ VARIABLES DE EMPODERAMIENTO } \\
\hline & \multicolumn{2}{|c|}{ GÉNERO } & \multicolumn{2}{|c|}{ CADENA DE VALOR } & \multirow{2}{*}{ Total } \\
\hline & Agencia & Estructura & Desarrollo & Gobernabilidad & \\
\hline \multicolumn{6}{|l|}{$\begin{array}{l}\text { Mitigar la resistencia } \\
\text { a partir de la tradición }\end{array}$} \\
\hline $\begin{array}{c}\text { Formalizar las cadenas de } \\
\text { valor de mujeres rurales }\end{array}$ & 3 & 2 & 2 & 3 & 10 \\
\hline $\begin{array}{c}\text { Convertir responsabilidades } \\
\text { tradicionales en nuevas } \\
\text { oportunidades }\end{array}$ & 2 & 2 & 2 & 2 & 8 \\
\hline $\begin{array}{l}\text { Transformar los sistemas } \\
\text { tradicionales a través de } \\
\text { nuevos roles para las mujeres }\end{array}$ & 2 & 2 & 2 & 2 & 8 \\
\hline $\begin{array}{l}\text { Mejorar la participación de } \\
\text { las mujeres por medio de } \\
\text { procesos agroecológicos }\end{array}$ & 4 & 4 & 4 & 4 & 16 \\
\hline \multicolumn{6}{|l|}{$\begin{array}{c}\text { Crear espacios } \\
\text { para las mujeres }\end{array}$} \\
\hline $\begin{array}{l}\text { Posicionar y comprometer } \\
\text { a las mujeres en cadenas } \\
\text { dominadas por hombres }\end{array}$ & 4 & 3 & 3 & 3 & 13 \\
\hline $\begin{array}{l}\text { Fomentar iniciativas } \\
\text { empresariales femeninas }\end{array}$ & 2 & 2 & 2 & 2 & 8 \\
\hline \multicolumn{6}{|l|}{ Organizar para cambiar } \\
\hline $\begin{array}{c}\text { Estimular el desarrollo } \\
\text { de capacidades }\end{array}$ & 4 & 3 & 3 & 2 & 12 \\
\hline $\begin{array}{c}\text { Desarrollar acciones } \\
\text { colectivas }\end{array}$ & 4 & 2 & 3 & 2 & 11 \\
\hline $\begin{array}{c}\text { Sensibilizar el pensamiento } \\
\text { masculino }\end{array}$ & 2 & 2 & 2 & 2 & 8 \\
\hline $\begin{array}{l}\text { Gestionar financiamiento de } \\
\text { cadenas de valor para mujeres }\end{array}$ & 2 & 2 & 2 & 2 & 8 \\
\hline \multicolumn{6}{|l|}{$\begin{array}{c}\text { Estándares, certificación } \\
\text { y etiquetas }\end{array}$} \\
\hline $\begin{array}{c}\text { Crear etiquetas y sellos } \\
\text { comercializando la } \\
\text { participación de las mujeres }\end{array}$ & 3 & 3 & 2 & 2 & 10 \\
\hline $\begin{array}{l}\text { Aprovechar estándares } \\
\text { y sistemas de } \\
\text { certificación existentes }\end{array}$ & 2 & 2 & 2 & 2 & 8 \\
\hline $\begin{array}{l}\text { CRITERIOS DE } \\
\text { CALIFICACIÓN }\end{array}$ & $1 \mathrm{Ine}$ & tente 2 Insuf & ciente 3 Regu & ar 4 Bueno 5 Excel & \\
\hline
\end{tabular}

Fuente propia. 
tradicionales en nuevas oportunidades" y "transformar los sistemas tradicionales a través de nuevos roles para las mujeres". Esto ratifica la posición de Ferreiray Mattos (2017), quienes plantean la agroecología como estrategia potencial para abrir espacios donde las mujeres rurales enfrenten su condición de vulnerabilidad y puedan desarrollar esa capacidad y poder de acción en el ámbito personal, productivo, familiar y político.

En segundo lugar, está la dimensión "crear espacios para las mujeres", cuyas, alternativas de empoderamiento femenino, incorpora a las mujeres en cadenas comúnmente dominadas por hombres para incrementar su visibilidad y poder de decisión a nivel económico. Además, estimula el emprendimiento de las mujeres, facilitando la creación de nuevas empresas o el mejoramiento de las ya existentes (Senders, et al. 2015). En este sentido, la alternativa de "posicionar y comprometer a las mujeres en cadenas dominadas por hombres", ha obtenido una mayor calificación que "fomentar iniciativas empresariales femeninas". Esto quiere decir que la segunda es consecuencia de la primera y considerando que ya hay un trabajo previo, donde la capacidad de asumir nuevos roles ha aumentado, es posible asumir compromisos que promuevan el cambio de rol dentro de la cadena, sin necesidad de crear un nuevo emprendimiento.

En tercer lugar, la dimensión "organizar para cambiar", contiene alternativas para empoderar a las mujeres en la parte superior de la cadena a través de procesos de organización enfocados al cambio dentro del hogar, de la producción y de su posición dentro de la cadena (Senders, et al. 2015). Para ello debe estimular el desarrollo de las capacidades, fortaleciendo la confianza, autoestima y habilidades de las mujeres rurales. Seguidamente se debe desarrollar acciones colectivas que permitan trabajar en los mecanismos de acceder a asistencia técnica y servicios de extensión, buscando alcanzar productividad, eficiencia y valor agregado. Como resultado, se obtendrá la sensibilización del pensamiento masculino, promoviendo la trasformación de los roles de género y la división equitativa del trabajo entre hombres y mujeres. Esto conlleva a la gestión del financiamiento de cadenas de valor, permitiendo el desplazamiento de las productoras de su rol como actores básicos a copropietarias de la cadena de valor agrícola.

Por último, la dimensión "estándares, certificación y etiquetas", será ese gran resultado y prueba de empoderamiento de las mujeres rurales, cuando se logre crear etiquetas y sellos que comercialicen la participación de las mujeres, dando el reconocimiento al esfuerzo, dedicación y significativo aporte al desarrollo del sector agrícola, con lo cual se ratifique la importancia de empoderar a los productores de pequeña escala para la sostenibilidad. Mujeres en distintos lugares y espacios de producción, consumo, comercialización, movilización política o generación de conocimiento se van involucrando y visibilizando de manera comprometida y sistemática, superando barreras condicionadas al rol de género y conviviendo en un ambiente de equidad (Zuluaga, Catacora y Siliprandi. 2018). 
En resumen, las alternativas de mayor importancia a nivel general son: mejorar la participación de las mujeres por medio de procesos agroecológicos (16 puntos), posicionar y comprometer a las mujeres en cadenas dominadas por hombres (13 puntos), estimular el desarrollo de capacidades (12 puntos) y crear etiquetas y sellos comercializando la participación de las mujeres (10 puntos). Estas constituyen el camino a seguir y en la medida que avance el proceso de empoderamiento y participación de las mujeres rurales, se podrán implementar las alternativas restantes según el valor de priorización.

\section{Equidad de género para el desarrollo rural}

Para Duarte y García (2016), la equidad de género requiere que todas las dimensiones de la justicia incorporen la perspectiva de género en los documentos legislativos, con el objetivo de combatir las discriminaciones, la desigualdad, la opresión y la violencia contra hombres y mujeres; esto implica imparcialidad en el trato para mujeres y hombres, según sus respectivas necesidades, a fin de contar con los mismos beneficios y ejercer sus derechos. Según estos autores, al hablar de igualdad de género, se refiere a que la mujer y el hombre tienen igual libertad para la realización de sus derechos humanos, desarrollar sus capacidades personales y elegir sin limitaciones establecidas por estereotipos para ejercer su potencial de contribución al desarrollo político, económico, social, cultural y de disfrutar de los resultados. Así que todo comportamiento, aspiración y necesidad de cada uno sea valorado, considerado y favorecido de la misma manera independientemente de su rol de género (KIT, Agri-ProFocus and IIRR, 2013).

Dada la necesidad de exigir justicia social por parte de las mujeres, surge el concepto de feminismo, entendido como una ideología y un conjunto de colectivos políticos, culturales y económicos que tienen como objetivo la igualdad de los derechos de las mujeres y de los hombres (Duarte y García. 2016). La teoría de género y el feminismo, tiene como sinónimos: enfoque de género, visión de género, mirada de género, etc. El enfoque de género permite analizar cómo las diferencias biológicas se convierten en desigualdades sociales y colocan a las mujeres en desventajas respecto a los hombres. De igual manera, permite ver cómo se construyen, sostienen y reproducen mediante una serie de estructuras sociales y mecanismos culturales qué cambian a lo largo del tiempo en los espacios (Flores y Nayeli, 2015). En este sentido, surge una serie de tendencias entre las que se destaca el ecofeminismo, pertinente para el análisis de este estudio de caso.

En este marco, las relaciones de género también son formas de apropiación, distribución, acceso y uso del territorio. Entender estas relaciones intrínsecas es importante debido a que las mujeres son portadoras de conocimientos y prácticas del manejo de los recursos naturales que resultan claves y deben ser tomados en cuenta si se busca promover una gestión de desarrollo viable, sostenible y socialmente incluyente. Entre estas relaciones, el ecofeminismo marca el proceso de evolución 
que ha tenido la vinculación entre mujer y ambiente, teniendo como horizonte común el definir un nuevo concepto de desarrollo y superar las inequidades, la degradación y sobreexplotación que también se hace manifiesta en la mujer (Ricaldi, 2004).

Desde esta perspectiva, se abordan otros conceptos, como es el caso del desarrollo rural, que abarca diversos factores y está orientado a mejorar la calidad de vida de las poblaciones, dejando atrás la visión sectorial y reduccionista del campo, en este confluyen otros enfoques como el desarrollo local, las nuevas ruralidades, la multifuncionalidad del espacio rural y el capital social (Márquez y Foronda, 2005). El desarrollo rural con enfoque territorial se asienta en el supuesto de una multiplicidad de acciones de tipo económico-productivas e institucionales, promovidas por actores de diversos sectores que interactúan en un territorio, cooperan y se articulan con actores locales, cuya sinergia converja en un todo, de acuerdo con las concepciones neo-institucionalistas que reivindican la importancia del capital social (Gonzales, Gaviria y Cabezas, 2019).

Tomando como referencia los anteriores conceptos, se analiza desde la experiencia de las mujeres emprendedoras de San Roque, el proceso paulatino que han venido adelantando en la incorporación de criterios de equidad de género para el fortalecimiento de cadenas de valor agrícola. Es de rescatar el interés de estas mujeres rurales por reclamar un trato equitativo respecto a los beneficios que los hombres reciben, principalmente lo relacionado a las oportunidades de financiación, empoderamiento económico y reconocimiento social. Con ello, dan los primeros pasos para exigir equidad de género. Así mismo, han adelantado acciones de organización comunitaria de manera libre y espontánea, con las cuales han fortalecido sus capacidades personales y a pesar de las limitaciones culturales y políticas han adelantado procesos organizativos en beneficio común.

Este hecho se ha ido replicando a nivel de todo el corregimiento de Chapa, ya que en el sector, ASMESAR fue la primer asociación conformada por productores agropecuarios y la primera organización de mujeres rurales, quienes con su deseo de mejorar sus ingresos económicos, han promovido prácticas agroecológicas y acciones en pro de la conservación de los recursos naturales. A la fecha, ya se cuenta con cuatro asociaciones que lideran proyectos productivos agropecuarios y en ecoturismo, esto se traduce en una primera contribución al desarrollo rural de origen comunitario impulsado por una iniciativa de mujeres rurales en el territorio.

Hablar desde la perspectiva de género a nivel comunitario y de políticas públicas, incorpora el concepto de igualdad de oportunidades y derechos a partir de la identificación de necesidades y demandas diferenciadas que tienen hombres y mujeres en todas las instancias de acción del Estado al tiempo que se avanza en la igualdad de género. En consecuencia, los responsables de la definición, diseño, implementación, seguimiento y evaluación de la política pública asumen el compromiso de superar 
las desigualdades de género y toman en cuenta de manera sistemática los efectos diferenciados que ejercen las acciones de cada sector en mujeres y hombres (Malcolm, 2018).

\section{Conclusiones}

El fortalecimiento asociativo integral enfocado a la soberanía alimentaria de la población a través de la agroecología, permite que las mujeres rurales impulsen la transformación del rol que tradicionalmente vienen desempeñando a nivel familiar con proyección a la comunidad. Esta dinámica aporta a las mujeres rurales mayor capacidad para la negociación al interior de la familia, dado a que genera cambios en la redistribución de tareas al interior del hogar y la corresponsabilidad asumida por toda la familia, al producir y gestionar parte de los ingresos. Tal autonomía reflejada en el caso de estudio de las mujeres rurales de la Vereda San Roque impacta positivamente, porque se evidencia el empoderamiento y liderazgo, fundamentales en la toma de decisiones, además, modifican las condiciones de género que imposibilitaban el desarrollo de las capacidades humanas de las asociadas.

El desarrollo y fortalecimiento de cadenas de valor con enfoque de género es una estrategia que contribuye en la reducción de la alta vulnerabilidad de las mujeres rurales, siempre y cuando, estas ofrezcan a cada uno de los actores de la cadena, la posibilidad de desempeñar todos los roles dentro de esta, a partir del fortalecimiento de la capacidad de asumir posiciones de mayor liderazgo para la participación y toma de decisiones.

En síntesis, la incorporación de criterios de equidad de género en las cadenas de valor agrícola es viable para las mujeres, la sociedad y las actividades productivas. Una vez se facilite el acceso a recursos técnicos y económicos a las mujeres rurales, se disminuye las restricciones que limitan su desempeño, genera empoderamiento social y económico de la mujer. Esto favorece la mejora de la calidad de vidayla de su entorno, permitiendo transformaciones sostenibles alargo plazo, contribuyendo a un crecimiento económico, mejorando la gestión y desarrollo rural para las comunidades. 


\section{Agradecimientos}

El presente artículo de investigación es producto de la participación en el seminario "Agroecología y desarrollo territorial" del programa de Ecología de la Fundación Universitaria de Popayán (FUP) y la vinculación a la Asociación de Mujeres Emprendedoras de San Roque del municipio de Sotará ASMESAR, a quienes se manifiesta sinceros agradecimientos por la oportunidad de conocer su experiencia exitosa de asociatividad y compartir espacios de conocimiento en el desarrollo de procesos de empoderamiento comunitario. Igualmente, se agradece el acompañamiento y contribución en la formación académica en la construcción del artículo de los profesionales adscritos a los grupos de investigación UNIET de la FUP, GITA de Corporación Autónoma del Cauca y el grupo de Investigación del SENA.

\section{Bibliografía}

Acosta, B. V., Zambrano, M., Suárez, S.M. 2017. Emprendimiento femenino y ruralidad en Boyacá, Colombia. Criterio Libre. vol. 15(26): 215-236. https://revistas.unilibre.edu.co/index.php/ criteriolibre/article/view/1048.

Agüero, F. R., Gutiérrez, I. A., Hernández, L. H. Escobedo, A. y Salvador, E. 2012. El empoderamiento de las mujeres en las cadenas de valor: Un reto para las políticas de desarrollo rural. doi: 10.13140/2.1.4876.6088.

Altieri, M. A. 1999. Agroecología. Bases científicas para el desarrollo rural sustentable. Editorial Nordan-Comunidad, Montevideo. $325 \mathrm{pp}$.

Casique, I. 2010. Factores de empoderamiento y protección de las mujeres contra la violencia. Revista Mexicana de Sociología. vol. 72(1): 37-71. [http://www.scielo.org.mx/scielo. php?script=sci_arttext\&pid=So188-25032010000100002. 
Cediel, N. y Morales, P. 2019. Equidad de género en la tenencia y control de la tierra en Colombia: llamado a una acción emancipadora. Revista. Medica. Veterinaria. vol. 1(37): 7-12. doi: 10.19052/mv.vol1.iss37.1.

Cortínez, V. 2016. Igualdad de género para el desarrollo territorial: experiencias y desafíos para América Latina. Serie documentos de trabajo. vol. 180. p. 22, http://www.americalatinagenera.org/ newsite/images/Cortinez2o16.pdf.

Desarrollo de cadenas de valor sensibles al género. [Internet] 2017. Organización de las Naciones Unidas para la Alimentación y la Agricultura - FAO. Fecha de acceso: 2020 Abr 10. Disponible en: http://www.fao.org/documents/card/en/c/ig212es

Díaz Pérez,Á. M., \& Silva Niño, A. C. [Internet] 2019. Modelo de empoderamiento socioeconómico con enfoque de género: La experiencia de Corambiente con mujeres rurales en Santander. https://repository.unab.edu.co/handle/20.500.12749/10727

Duarte,J. M. y García,J. B. 2016. Igualdad, Equidad de Género y Feminismo, una mirada histórica a la conquista de los derechos de las mujeres. Rev. CS, vol. 18: 107-158. doi: 10.18046/recs.118.1960.

Estébanez, M. E., Sued, G. E., Turkenich, M. y Nicosia, S. F. 2016. Género e innovación en la producción agrícola de baja escala. Rev. Iberoamericana Ciencia, Tecnología y Sociedad, vol. 11: 217-246. http://www.revistacts.net/volumen-11-numero-31/313dossier/713-genero-e-innovacion-en-la-produccion-agricolade-baja-escala.

Flores R. y Nayeli, L. 2015. El enfoque de género y el desarrollo rural: ¿necesidad o moda?, Rev. Mex. Ciencias Agrícolas, 1 : 401-408. https://www.redalyc.org/pdf/2631/263139243054.pdf.

González, V. E., Gaviria A. G. y Cabezas, F. 2019. El trabajo productivo y la asociatividad de la mujer rural conceptuada desde el empoderamiento económico y la perspectiva de género. Estudio de caso en la Asociación de Mujeres Caficultoras 'MAIKAFE' Municipio de Piendamó, Departamento del Cauca- 
Colombia. Tesis de maestría en Gestión de las Organizaciones. Universidad Cooperativa de Colombia. Facultad de Ciencias Administrativas, Económicas y Contables. Popayán. 107 pp. https://repository.ucc.edu.co/handle/20.500.12494/7851

Grant, M.J. and Booth,A. 2009. A typology of reviews: An analysis of 14 review types and associated methodologies. Health Info. Libr.J., 26(2): 91-108. doi: 10.1111/j.1471-1842.2009.00848.x.

Güiza, L., Rodríguez, C. D., Ríos, B. O B., y. Moreno, S. S. 2016. Género y empoderamiento comunitario en un contexto de posconflicto: el caso de Vergara, Cundinamarca (Colombia). Estud. Socio-Jurídicos, vol. 18(2):115-144. doi: 10.12804/ esj18.02.2016.04.

Kim, S. M. and Sherraden, M. 2014. The impact of gender and social networks on microenterprise business performance.J. Sociol. Soc. Welf., vol. 41(3):48-70. https://www.researchgate.net/ publication/305317486_The_Impact_of_Gender_and_Social_ Networks_on_Microenterprise_Business_Performance.

Lagarde, R. M.1996. Género y feminismo. Desarrollo humano y Democracia. Horas y Horas. 13-38. https://catedraunescodh. unam.mx/catedra/CONACYT/o8_EducDHyMediacionEscolar/ Contenidos/Biblioteca/Lecturas-Complementarias/Lagarde_ Genero.pdf

Lamas, M. 200o. Diferencias de sexo, género y diferencia sexual. Escuela Nacional de Antropología e Historia (ENAH), 7(18): 1-25. http://repositorio.ciem.ucr.ac.cr/jspui/handle/123456789/157

Las mujeres rurales y la agricultura familiar. [Internet] 2015. Organización de las Naciones Unidas para la Alimentación y la Agricultura - FAO. Fecha de acceso: 2020 May 25. Disponible en: https://www.minsalud.gov.co/sites/rid/Lists/ BibliotecaDigital/RIDE/VS/PP/SNA/Boletin-01-2015-Mujeresrurales-agricultura-familiar.pdf.

Malcolm, C. M. 2018. Perspectiva de género y desarrollo rural. Análisis de la implementación local a través de un estudio 
de caso. Tesis Maestría en Administración y Política Pública. Universidad de San Andrés. Departamento de Ciencias Sociales. Buenos Aires. 131 pp. http://hdl.handle.net/10908/16023

Marmolejo, G. A., Godoy, M. A y González, C. C. 2017. De amas de casa a mujeres empresarias. Reseña de una empresa rural guanajuatense liderada por mujeres, 2(2): 1-13. https://biblat. unam.mx/hevila/UPGTOmanagementreview/2017/vol2/no2/5.pdf

Márquez, D. y Foronda, C. 2005. El capital social eje del desarrollo en espacios rurales. Cuaderno de Geografía, vol. 78:155-176. http://dialnet.unirioja.es/servlet/articulo?codigo $=2363578$.

Martínez, P. 2011. El método de estudio de caso Estrategia metodológica de la investigación científica. Revista científica Pensamiento y Gestión, (20). http://rcientificas.uninorte.edu.co/ index.php/pensamiento/article/view/3576/2301

Medina, J., Ortiz, F., Franco, C. y Aranzazú, C. 2010. Matriz de Priorización para la Toma de Decisiones. Facultad de Ciencias de la Administración Universidad del Valle. Cali, Colombia, pp. 1-23. http://sigp.sena.edu.co/soporte/Plan/o3_Matriz de priorización.

Moreno,J. 2015. Participación y empoderamiento de las mujeres rurales y su aporte para mejorar las condiciones de pobreza y diseño de políticas públicas con equidad de género. Universidad Pablo de Olavide de Sevilla, Tesis Doctoral en Economía. Sevilla. 26o pp. https://rio.upo.es/xmlui/handle/10433/2372

Nobre, M., Hora, K., Brito, C. y Parada, S. 2017. Atlas de las mujeres rurales de América Latina y el Caribe: "Al tiempo de la vida y los hechos.”. Organización de las Naciones Unidas para la Alimentación y la Agricultura (FAO). 82 pp. http://www.fao. org/3/a-i7916s.pdf

Nuestras prioridades: Los objetivos estratégicos de la FAO. [Internet] 2013. Organización de las Naciones Unidas para la Alimentación y la Agricultura - FAO. Fecha de acceso: 2020Jun 2. Disponible en: http://www.fao.org/3/mi317s/mi317s.pdf. 
Palomar, C. 2016. Veinte años de pensar el género. Debate Femenino, vol. 52:34-49. doi: 10.1016/j.df.2016.09.002.

Ricaldi, T. 2004. La equidad de género en las políticas de desarrollo: un desafío para la sustentabilidad. Polis. Revista Latinoamericana, 9:1-17. http://journals.openedition.org/ polis/ 7258

Rodríguez, Y. 2018. Empoderamiento de mujeres rurales del sur del Cauca: Un caso regional de innovación social. Universidad ICESI, [Internet]. Fecha de acceso: 2020 May 26. Disponible en: https://repository.icesi.edu.co/biblioteca digital/bitstream/10906/84385/1/To1477.pdf

Royal Tropical Institute (KIT), Faida MaLi and IIRR. 2006. Chain empowerment: Supporting African farmers to develop markets. Royal Tropical Institute, Amsterdam; Faida Market Link, Arusha; and International Institute of Rural Reconstruction, Nairobi. http://www.mamud.com/Docs/chains.pdf

Royal Tropical Institute, (KIT), Agri-ProFocus, and (IIRR) International Institute of Rural Reconstruction. 2012. "Challenging chains to change."

Royal Tropical Institute (KIT), Agri-ProFocus and International Institute of Rural Reconstruction IIRR. 2013. Mujeres al frente. Equidad de género en el desarrollo de la cadena de valor agrícola. Amsterdam. https://agriprofocus. com/upload/mujeres_al_frente_-_chachacha_espanol. compressed1429612926.pdf

Segovia, D. 2009. Agroecología y Desarrollo Sustentable. Debates para la acción. BASE Investigaciones Sociales. Documento de trabajo $\mathrm{N}^{\circ}$ 128: 44pp. [Internet]. Fecha de acceso: 2020 Abr 3. Disponible en: http://www.baseis.org.py/ wp-content/uploads/2014/03/139515508o.pdf

Senders, A., Lentink, A., Vanderschaeghe, M., Terrillon, J., Snelder, R. y Agri-ProFocus. 2015. Género en cadenas de valor, 
Caja de herramientas prácticas para integrar una perspectiva de género en el desarrollo de cadenas de valor agropecuarias.”, [Internet]. Fecha de acceso: 2020 Abr 3. Disponible en: https:// agriprofocus.com/upload/FINAL-Caja_Herramientas. compressed1415291170.pdf.

Sicard, T. L. 2010. Capítulo 2: Agroecología: desafíos de una ciencia ambiental en construcción, p.57. En: Sicard, Tomas León y Altieri, Miguel Ángel (2010). Vertientes del pensamiento agroecológico, Fundamentos y Aplicaciones. Ideas 21, SOCLAIDEA, 293p.

Terrillon, J. 2010. Gender Mainstreaming in Value Chain Development: Practical guidelines and tools [Internet]. Fecha de acceso: 2020 Jun 24. Disponible en: http://www.fao.org/3/aat227e.pdf.

The state of agriculture and food. [Internet] 2017. Organización de las Naciones Unidas para la Alimentación y la Agricultura FAO. Fecha de acceso: 2020 Jul 1. Disponible en: http://www.fao. org/3/a-i7658s.pdf

Toledo, V. M. 2011. Del "diálogo de fantasmas" al "diálogo de saberes": conocimiento y sustentabilidad comunitaria. Pp 469-484. En: Argueta, A., Corona, E. y Hersch, P. (Ed.) Saberes colectivos y dialogo de saberes en México. Universidad Nacional Autónoma de México. Centro Regional de Investigaciones Multidiciplinarias Universidad Iberoamericana/Puebla. Cuernavaca, Morelos. 574 pp. Disponible en: https://www.crim. unam.mx/web/sites/default/files/Saberes\%2ocolectivos\%20y\%20 di\%C3\%A1logo\%2ode\%2osaberes.pdf

Zarate, M. 2019. El efecto delabrecha de género sobrela agricultura y la soberanía alimentaria en las comunidades indígenas del occidente de Guatemala. Tesis Maestría en Cooperación al desarrollo. UniversitatJaume. Guatemala. 67 pp. http://repositori. uji.es/xmlui/bitstream/handle/10234/186890/TFM_2019_ Zarate\%2oHerrero_\%2oMaitane.pdf?sequence=1\&isAllowed=y 
Zuluaga Sánchez, G. P., Catacora-Vargas, G., \& Siliprandi, E. 2018. Agroecología en femenino. Reflexiones a partir de nuestras experiencias. SOCLA-CLACSO. https://www. researchgate.net/publication/328732640_Agroecologia_en Femenino_Reflexiones_a_partir_de_nuestras_experiencias 